\title{
Minimal Standards for Electroencephalography in Canada
}

\author{
Prepared by a Task Force* of The Canadian Society of Clinical \\ Neurophysiologists
}

Can. J. Neurol. Sci. 2002; 29:216-220

More than a decade has passed since the publication of the last document of national electroencephalography (EEG) standards (Young 1991). Since then, advances in EEG technology, the formulation of national technology guidelines and more detailed evaluations of EEG procedures, findings and indications have transpired. Hence, a Task Force of the Canadian Society of Clinical Neurophysiologists (CSCN) was struck to revise the standards for the practice of EEG in Canada. The Task Force's document was prepared and revised following consultations from numerous clinical neurophysiologists and EEG technologists from across the country and was finally accepted at the CSCN annual general meeting in June of 2001. It is intended to serve as a guide for EEG laboratories but is not meant to be permanent or all-inclusive. Electroencephalographers and technologists are encouraged to utilize other quality standards documents and papers (see references) and to keep abreast of the literature.

Our guideline development gave primacy to the ancient medical principle, "first, do no harm". That is, EEG must be safe; there should not be a risk from infectious or electrical hazards or other potential dangers.

EEG must provide reliable information. Electroencephalograms should be performed in an accurate, careful and responsible manner by technologists who are competent, using adequate equipment. Then, competent electroencephalographers carefully and promptly report the results. The report contains a description of the characteristics of the recording. The description is followed by a clinical interpretation or conclusion that correlates the clinical features with the findings on the EEG tracing.

\section{A. Electroencephalographers}

\section{Standards for Electroencephalographers}

The only national standard for electroencephalographers is the qualifying examination developed by the EEG section of the CSCN. The CSCN strongly recommends that provinces that do not have a provincial qualifying examination use the successful passing of this examination as a prerequisite for the practice of electroencephalography. This should especially apply to physicians who are beginning to practice. During the implementation phase, a "grandfathering" qualification is usually needed to allow competent electroencephalographers who are already in established practice to continue. All electroencephalographers should have the following qualifications:

(a) the individual should be a licensed physician within the province of practice and should have a fellowship certificate of the Royal College of Physicians and Surgeons of Canada or a $\mathrm{PhD}$ in a basic science related to the field of EEG. The latter individuals should have distinguished themselves by their competence as clinical interpreters.

(b) he/she should have completed a minimum of six months of full time supervised training in a laboratory that is run by a full member of the CSCN or of the American Clinical Neurophysiology Society.

\section{Laboratory Director}

Each EEG laboratory should appoint a director who is a physician who meets the CSCN qualifications for the practice of EEG. Responsibilities of the director should include the following:

- day-to-day functioning of the laboratory,

- ensuring a quality assurance or quality improvement program is in place,

- selection of testing procedures to be used in recordings,

- making available for accreditation the requested documentation,

- providing continuous, adequate and effective direction and supervision of the laboratory staff.

\section{Locum Tenens}

A locum tenens is defined as a medical practitioner who undertakes the professional duties of another medical practitioner during the latter's absence.

It is strongly recommended that a locum tenens who functions in an acute hospital setting or independent clinic should possess the same qualifications as listed above for electroencephalographers.

\section{Continuing Education}

It is recommended that continuing educational activities be in keeping with the Maintenance of Certification Program of the Royal College of Physicians and Surgeons of Canada. Individual regular review of journals and attendance at local conferences are important. It is recommended that the electroencephalographer regularly meet with the technologists for reading sessions

\footnotetext{
* Task Force Members: R. Arts, C. Blackburn, D. Brunet, W. Ilse, J. Maher, A. Ogunyemi, E. Starreveld and B. Young.

ReCEIVEd February 6, 2002. ACCEPTEdin finalform May 6, 2002. Reprint requests to: Canadian Society of Clinical Neurophysiologists, 709 - 7015 Macleod Trail SW, Calgary, Alberta T2H 2K6 Canada
} 
and, if possible, other electroencephalographers in the area. It is strongly recommended that the electroencephalographer regularly attend national or international scientific meetings such as the Canadian Congress of Neurological Sciences, American Clinical Neurophysiology Society, the American Epilepsy Society, the American Academy of Neurology or refresher courses at other meetings on EEG and epilepsy.

\section{B. ELECTROENCEPHALOGRAPHIC TECHNOLOGISTS}

The Canadian Board of Registration of Electroencephalographic Technologists, Inc. (CBRET) is the only national organization that provides a qualifying examination. The CSCN recommends passing the CBRET examination as the national standard for qualification as an EEG technologist. It is recognized that provincial/regional authorities have the final authority in setting standards for EEG technologists for that province/region. Furthermore:

1. Each EEG laboratory should have a minimum of one provincially-accredited EEG technologist, usually a CBRETregistered technologist. In special circumstances, exceptions could be made at the discretion of the reviewing body within each province.

2. Electroneurodiagnostic training programs should be in laboratories run by a member of the EEG section of the CSCN with at least one CBRET-registered technologist.

3. Continuing Education: it is suggested that medical directors of clinical EEG laboratories promote continuing education for their laboratory staff and assist them in successful completion of Board examinations. In addition, regular reading sessions and regular attendance at provincial, Canadian and international meetings are encouraged. Funding for such continuing education should reside with the health facility. It is recommended that EEG technologists have membership in the provincial technologist organizations and the Canadian Association of Electroneurophysiology Technologists (CAET).

4. Cardiopulmonary resuscitation (CPR): it is strongly recommended that technologists be certified in CPR and recertified every two years.

\section{LABORATORY STANDARDS AND PROCEDURES: EEG EQUIPMENTAND RECORDING TECHNIQUES}

1. In a clinical setting at least $\mathbf{1 6}$ channels of EEG should be recorded, unless there are exceptional circumstances such as scalp lesions, bandages, intensive care unit (ICU) settings or limited strip/grid recordings. Recordings should be for at least 20 minutes. Routine recordings should include hyperventilation for at least three minutes and photic stimulation at various flash frequencies with the eyes open and closed, unless the medical condition does not allow this. Photic stimulation can be omitted in neonates and in the very old patient.

Montages should be designed for adequate spatial sampling; it is recommended that most montages include over $70 \%$ of the electrodes of the International 10-20 System of Electrode Placement. A single montage with at least eight channels of EEG electrodes from the frontal, temporal, central, occipital and midline regions, along with additional channels that should include the electrocardiogram (EKG), electro-oculogram (EOG), electromyogram (EMG) and respirations, is recommended in the neonatal period.

It is desirable to obtain both awake and sleep recordings. Neonatal EEGs should be interpreted at a slower paper speed (e.g., $15 \mathrm{~mm} / \mathrm{sec}$ ) for at least part of the recording. A full hour (60 minutes) of recording is recommended.

The technologist should take a history from the patient and record this with any relevant observations before, during or after the recording. Any changes in patient state should be noted.

\section{Infection Control}

a. Needle electrodes must not be used in routine clinical settings and never used in out-of-hospital laboratories. If exceptional clinical circumstances, e.g., burns to the head or certain intraoperative procedures, require their use, sterilized, single-use needle electrodes are used and disposed of after the recording.

b. In patient preparation, the use of blunt tip needles is not recommended. To reduce skin impedance, the use of abrasive gels applied with a cotton-tipped applicator is preferred. However, if the clinical situation warrants the use of such needles for lowering impedances (e.g., there is difficulty in adequately reducing the impedance), sterilized disposable blunt tip needles must be used for each patient and discarded after each use.

c. Appropriate cleaning, disinfecting and/or sterilization of electrodes and accessories must be carried out between patients. These should meet the standards of the provincial College of Physicians and Surgeons. Fabric fitted caps with embedded electrodes should be used with discretion and caution, because of difficulties in cleaning them without damaging the apparatus. They should not be used in patients with breaks in the skin, wounds, burns or known or potentially transmissible diseases, including infectious hepatitis, human immunodeficiency virus or CreutzfeldtJakob disease.

d. For ill in-patients, the technologist should consult with the nursing staff concerning any special infection control requirements for the patient.

\section{Digital/Quantitative EEG}

Digitial EEG offers many advantages over paper-based technology, including the opportunity to reformat montages, to apply and remove filter settings and automated detection strategies and to perform longer term recordings. Although in some ways, the technologists' duties are simplified, the same rigor of electrode application, observation and annotation is required.

Optical disk, compact disk read-only-memory (CD-ROM) and digital video disk (DVD) technology are acceptable media for storage of digital EEG recordings.

To assure the adequacy of waveform recording a minimum sampling rate of 200 samples per second for each channel should be used. Digitization at voltage level of 12 bits or greater, to resolve voltage to $0.5 \mu \mathrm{V}$, is recommended. Common mode rejection should be $100 \mathrm{~dB}$ or greater at each amplifier output. Interchannel cross-talk must be less than $1 \%$, i.e., $40 \mathrm{~dB}$ down or less.

The available technology should allow for the display of the recording on a video screen as well as on paper. It is recommend that, with horizontal scaling, one second of time occupy $25-35 \mathrm{~mm}$ 
and contain at least 120 data points per channel; scaling at $0.5,2$ and 4 times should be feasible. On vertical display, a minimum spacing of $10 \mathrm{~mm}$ between channels for a display of 16 or 18 channels is recommended. Adequate screen resolution is at least 4 pixel resolution per vertical millimetre. It is recommended that the screen have at least 1024 x 768 pixels. Playback systems should show montage, filter settings, vertical voltage scale, horizontal time scale, technologist's comments, event markings, and page number or time. The playback unit should allow for post-hoc selection of montages, filters and sensitivity.

Topographic mapping, frequency or power spectral analyses and other quantitative assessments of digital EEG data should not be considered an alternative to traditional (standard) EEG display in either digital or analog systems. The interpretation should always involve interpretation of the standard EEG with quantitative methodologies being used to complement this. The use of quantitative EEG alone can yield misleading information when used in isolation.

\section{Electrical Standards}

Equipment should meet the minimal standards of Canadian Standards Association. Equipment should be purchased from nationally recognized manufacturers of electroencephalographs. If all components for a machine are not from the same manufacturer, the machine should be tested and approved by a qualified biomedical engineer before it is used. If this is not feasible, such a multi-component machine should not be used and a purchase instead be made from a single recognized electroencephalographic manufacturer.

Electrical safety in special care units: Equipment must be checked before each recording; current leakage should be checked at least twice yearly. Two-wire ungrounded devices, that are not essential to patient survival, should be unplugged, if agreed upon by attending staff. If needed, these instruments must be properly grounded and plugged into an isolation transformer. The electroencephalograph must be plugged into a three-wired grounded outlet located in the same circuit branch that is used to power the other instruments connected to the patient. Extension cords must not be used. The reference or common line from the patient must be electrically isolated from the power line ground or include an appropriate type of current-limiting device. No additional ground electrode should be used. When indwelling catheters or pacemaker electrodes are already connected to the patient, special precaution must be used to ensure that all these connections are properly isolated or current-limited. Switching the machine on and calibrating the electroencephalograph before the patient is connected and disconnecting the patient before switching off the instrument are recommended procedures. It is strongly recommended that electrical interference be promptly investigated, as this may indicate that there is current leakage through the patient.

\section{EEG Laboratory Manual}

The medical director has the ultimate responsibility for the policies and procedures laboratory manual. All procedures should be initially approved and signed by the medical director. The document should be reviewed annually by the director or his/her designate. The review should include the documentation of any changes made during the year. The manual should contain: a. Standards of practice documents.

b. Mission statement.

c. An information pamphlet for the patient.

d. An example of the EEG requisition and report form.

e. An organizational chart for the laboratory, including the chain of communication and responsibility, job descriptions, contact personnel and their telephone numbers.

f. Equipment-related material:

- a catalogue of equipment and age or time of purchase,

- maintenance contracts,

- preventive maintenance schedule,

- repairs,

- electrical checks,

- trouble shooting instructions for equipment problems.

g. Policies

- Policy for bookings: amount of time allotted per patient and the recording procedure including:

- head measurement technique: 10-20 system, application technique.

- policies for electrodes: types, cleaning techniques and disposal policy.

- policies for use of special techniques, indications and contraindications for activating procedures and sedation.

- recording protocol, including calibration.

\section{- Infection control policies:}

- Immunization policy: It is strongly recommended that all EEG technologists be immunized against serum hepatitis viruses.

- Safety measures and related techniques: It is recommended that technologists wear gloves when applying and removing electrodes or any other procedures on the head or body such as adjusting or moving electrodes or in altering their impedance. The use of two sets of gloves ("double gloving") is recommended when there is increased risk, e.g., performance of EEG on patients with serum hepatitis, acquired immune deficiency syndrome (AIDS), any dementing illness or with any active systemic infection. The principles of Standard Precautions and Transmission Based Procedures (formerly "universal precautions") should be respected.

- It is suggested that reinforcing statements be made concerning the avoidance of needle electrodes or the use of blunt-tip needles for skin abrasion.

- It is suggested that the manual contain procedures in the event of contamination with body fluids from a patient with possible transmissible infectious illness.

\section{EEG REQUISITIONS, RECORDS AND REPORTS}

\section{The EEG requisition should contain the following clinical details:}

The reasons for the test, medications the patient is taking, the clinical history, any special precautions (e.g., infection with human immunodeficiency or hepatitis viruses, allergies) any special requests for special procedures or special electrode 
positions. The requisition should be completed and signed by the referring physician prior to the test being done.

\section{Legal aspects of EEG records and reports}

Records should meet the guidelines for medical records as stated in the Public Hospitals Act for each province. In general, these should include:

a. Patient data documentation: patient's name, home address, date of birth, health number and a laboratory identification number. For hospitalized patients this should include a hospital identification number.

b. Documentation of the name of the referring physician.

c. Record and report storage: the storage of records and reports should follow provincial regulations for mandatory storage of patient records. Laboratories should retain a master list of EEG tests that includes all names of all patients tested, their identifying numbers and dates of tests.

\section{The EEG report}

\section{Guidelines:}

a. Factual information: Patient name, date of birth, other identifying information (e.g., hospital number), EEG number, clinical reasons for the test as per referring physician, relevant medications, state of patient (awake, asleep, coma, drug induced state), any variation from routine electrode positions or recording techniques that depart from the usual procedures of the laboratory (reasons should be given for departure from routine protocols), location of EEG if not in laboratory, special electrodes or techniques (e.g., sleep deprivation, sedative or other drugs used for the recording, drugs given during the recording), duration of recording if prolonged or if for electrocerebral silence.

b. Description of EEG activity: background rhythms and reactivity, normal and abnormal rhythms, normal and abnormal nonrhythmic phenomena, seizures, sleep (including staging), response to activating procedures: e.g., hyperventilation and photic stimulation, alerting stimuli. Also note artifacts if they interfere with reliable interpretation.

c. Clinical interpretation of EEG data and clinical information: This should include interpretation of the EEG normality or abnormality relevant to the clinical problem; correlation of the present with past recordings; diagnosis if apparent, or differential diagnoses. Appropriate feedback to the referring physician should be provided. The EEG technologist should convey only the electroencephalographer's official interpretation of the tracing to the referring physician's office and not his/her own interpretation. In special, isolated, emergency situations, the EEGer might choose to delegate the responsibility of providing EEG interpretation to the technologist. When this occurs, the EEGer should, as soon as possible, read the recording and provide confirmatory or additional feedback to the referring physician.

d. Classification system of EEGs: at the discretion of the laboratory director.

e. Timeline for relaying report: Routine EEGs should be reported in a timely fashion. Nonurgent EEGs usually are interpreted within five working days. It is recommended that the typed, signed report be available to the referring doctor within 10 days of the EEG. In routine cases the referring doctor would receive the report within at least two weeks of the procedure. If the results of the EEG suggest a medical emergency or a problem that needs urgent attention, the results should be promptly conveyed by telephone or some other means to the referring physician.

\section{E. INFORMED CONSENT}

All patients, or their substitute decision makers, should make an informed decision before having an EEG performed. For routine EEGs written consent is not necessary. The patient/ substitute decision maker should receive adequate information including the purpose of the EEG examination (being as specific as necessary), the nature of the test, the procedures used, and the potential risks and benefits. Competent patients should be informed that they have the right to consent to or refuse the test in whole or in part at any time during the procedure. When activating procedures (e.g., reduction of medications, sleep deprivation or the use of sedative drugs) place the patient at risk of seizures, excessive sedation or other complications, it is recommended that the patients or their caregivers be at least verbally informed of this.

It is strongly recommended that a fact sheet that gives the above-mentioned general information be utilized for elective EEGs.

In special situations, e.g., video recordings, invasive procedures (e.g., insertion of depth or subdural electrodes), written consent is strongly recommended.

\section{F.A DMINISTRATION OF SEDATION}

The administration of sedative drugs is seldom necessary for EEG recordings. Other methods of obtaining co-operation of the patient or of obtaining a sleep recording should be considered/attempted before sedative drugs are used. Before administering a sedative drug the risk/benefit ratio should be considered. It is recommended that the patient be screened for contraindications for administering the particular sedative drug, that informed consent be obtained, there is provision for adequate supervision and monitoring of the patient during the recording, that the patient be sufficiently awake and adequately protecting his/her airway before being discharged from the laboratory or facility.

\section{G. QUALITY IMPROVEMENT}

Periodic standardized patient and physician surveys are strongly recommended to be done by all EEG laboratories. Ideas that might be considered for inclusion in these surveys include:

1. waiting time for the test.

2. time from the test until report is received by the referring doctor.

3. the usefulness of the report to the referring doctor.

4. technical and professional treatment of the patients by the staff.

\section{REFERENCES}

Altman CL. Infection control 2000: Review and update for electroneurodiagnostic technologists. Am J END Technol 2000;40:73-97. 
American Electroencephalographic Society guidelines in electroencephalography, evoked potentials and polysomnography. J Clin Neurophysiol 1994;11:2-39, 88-115, 125-142.

Canadian Association Electroneurophysiology Technologists. Minimal Technical Standards: Electroencephalography, Electromyography, Evoked Potentials, Digital Electroencephalography, O.R. Monitoring EEG/EP, Infection Control, 2001.

Center for Disease Control and Epidemiology (CDC). Guideline for hand washing and hospital environmental control. Infect Control Hosp Epidemiol 1996;7:231-235.

Center for Disease Control and Epidemiology (CDC)/Hospital Infection Control Practices Advisory Committee (HICPAC). Guidelines for isolation precautions in hospitals. Am $\mathrm{J}$ Infec Control 1996;24:24-52.

Chatrian GE, Lettich E, Nelson PL. Ten per cent electrode system for topographic studies of spontaneous and evoked EEG activities. Am J EEG Technol 1985;25:83-92.

Hrachovy RA, Mizrahi EM, Kellaway P. EEG of the newborn. In: Daly DD, Pedley TA (Eds.) Current Practice of Clinical EEG. New York:Raven Press 1990;201-242.
Health and Welfare Canada. Blood borne pathogens in the health care setting: risk for transmission. Canada Communicable Disease Report 1992;18:177-184.

International Organization of Societies for Electrophysiological Technology (OSET). Guidelines for infection control in the clinical neurophysiology department. Am J END Technol 1999;39:289-300.

Jasper HH. The ten-twenty system of electrode placement of the International Federation. Electroenceph Clin Neurophysiol 1958; 10:371-373.

McLachlan RS, Young GB. Minimal standards for digital/quantitative electroencephalography in Canada. Can J Neurol Sci 1999;26:153.

Rutala WA. APIC guidelines for selection and use of disinfectants. Am J Infect Control 1990;18:99-117.

The College of Physicians and Surgeons of Ontario. Infection Control in the Physician's Office. May 1995.

Young GB. Minimal standards for electroencephalographic laboratories. CMAJ 1991;144:865-867. 